This is an electronic reprint of the original article. This reprint may differ from the original in pagination and typographic detail.

\author{
Author(s): Voss, Annika; Buchinger, F.; Cheal, B.; Crawford, J. E.; Dilling, J.; Kortelainen, Markus; \\ Kwiatkowski, A. A.; Leary, A.; Levy, C. D. P.; Mooshammer, F.; Ojeda, M. L.; Pearson, \\ M. R.; Procter, T. J.; Tamimi, W. Al
}

Title: Nuclear moments and charge radii of neutron-deficient francium isotopes and isomers

Year: $\quad 2015$

Version:

Please cite the original version:

Voss, A., Buchinger, F., Cheal, B., Crawford, J. E., Dilling, J., Kortelainen, M., Kwiatkowski, A.A., Leary, A., Levy, C. D. P., Mooshammer, F., Ojeda, M. L., Pearson, M. R., Procter, T. J., \& Tamimi, W. A. (2015). Nuclear moments and charge radii of neutron-deficient francium isotopes and isomers. Physical Review C, 91(4), Article 044307. https://doi.org/10.1103/PhysRevC.91.044307

All material supplied via JYX is protected by copyright and other intellectual property rights, and duplication or sale of all or part of any of the repository collections is not permitted, except that material may be duplicated by you for your research use or educational purposes in electronic or print form. You must obtain permission for any other use. Electronic or print copies may not be offered, whether for sale or otherwise to anyone who is not an authorised user. 


\title{
Nuclear moments and charge radii of neutron-deficient francium isotopes and isomers
}

\author{
A. Voss,${ }^{1,}{ }^{*}$ F. Buchinger, ${ }^{2}$ B. Cheal, ${ }^{3}$ J. E. Crawford,${ }^{2}$ J. Dilling, ${ }^{4,5}$ M. Kortelainen, ${ }^{1,6}$ A. A. Kwiatkowski, ${ }^{4}$ A. Leary, ${ }^{2}$ \\ C. D. P. Levy, ${ }^{4}$ F. Mooshammer, ${ }^{2}$ M. L. Ojeda, ${ }^{2}$ M. R. Pearson, ${ }^{4}$ T. J. Procter, ${ }^{4}$ and W. Al Tamimi ${ }^{2}$ \\ ${ }^{1}$ University of Jyvaskyla, Department of Physics, FI-40014 University of Jyvaskyla, Finland \\ ${ }^{2}$ McGill University, Department of Physics, Montreal, Quebec, Canada, H3A $2 T 8$ \\ ${ }^{3}$ University of Liverpool, Department of Physics, Liverpool, United Kingdom, L69 7ZE \\ ${ }^{4}$ TRIUMF, Vancouver, British Columbia, Canada, V6T $2 A 3$ \\ ${ }^{5}$ University of British Columbia, Department of Physics, Vancouver, British Columbia, Canada, V6T 1Z1 \\ ${ }^{6}$ Helsinki Institute of Physics, FI-00014 University of Helsinki, Finland \\ (Received 18 December 2014; revised manuscript received 12 March 2015; published 8 April 2015)
}

\begin{abstract}
Collinear laser fluorescence spectroscopy has been performed on the ground and isomeric states of ${ }^{204,206} \mathrm{Fr}$ in order to determine their spins, nuclear moments, and changes in mean-squared charge radii. A new experimental technique has been developed as part of this work which much enhances the data collection rate while maintaining the high resolution. This has permitted the extension of this study to the two isomeric states in each nucleus. The investigation of nuclear $g$ factors and mean-squared charge radii indicates that the neutron-deficient $\mathrm{Fr}$ isotopes lie in a transitional region from spherical towards more collective structures.
\end{abstract}

DOI: 10.1103/PhysRevC.91.044307

PACS number(s): 27.80.+w, 21.10.Ft, 21.10.Hw, 21.10.Ky

\section{INTRODUCTION}

Laser spectroscopic methods provide access to some of the most fundamental nuclear properties whereby both quantum (nuclear spin and magnetic dipole moment) and collective (electric quadrupole moment and changes in mean-squared charge radii) properties of the nuclei may be investigated. In the vicinity of the $Z=82, N=126$ shell closures, laser spectroscopy work using a variety of techniques has been applied [1-4].

By comparing the changes in mean-squared charge radii to isodeformation lines obtained from the droplet model [5,6], minimal deviations from zero deformation in the observed trend for the nuclear ground states in the $\mathrm{Pb}$ isotopic chain are observed from the spherical doubly magic nucleus ${ }^{208} \mathrm{~Pb}$ down to and beyond the neutron midshell at ${ }^{186} \mathrm{~Pb}$ [3]. Below the $Z=82$ shell closure in $\mathrm{Pb}$, odd-even staggering (OES) effects are observed around $N=104$ in studies of the neutrondeficient $\mathrm{Hg}$ isotopes [7]. The strong deviation from sphericity and the OES in the isotopic chains of $\mathrm{Hg}$ and, to a much lesser extent, $\mathrm{Pt}$ in the region near the midshell have been attributed to transitions between the different nuclear shapes $[8,9]$ caused by an intruder $\pi 1 h_{9 / 2}$ configuration. Both isotopic chains remain mostly spherical down to $N=106$.

Above the major shell gap in $\mathrm{Pb}$, the changes in meansquared charge radii in the Po isotopic chain show a gradual deviation from sphericity at $N=114$ caused by a shift in the dominant configurations from near-spherical to oblate [10]. The lack of current data in heavier even- $Z$ nuclei down to similar neutron numbers makes definitive statements difficult. However, the trend observed in the Rn $[2,11]$ seems to indicate a comparable effect with the onset of collectivity occurring at higher masses.

Typically, the neutron-deficient francium nuclei, having five protons in excess to $\mathrm{Pb}$, are considered to consist of an inert

*anvoss@jyu.fi
$\mathrm{Pb}$ core with valence nucleons around it [12]. As the neutron shells are depleted from $N=126\left({ }^{213} \mathrm{Fr}\right)$ downward, several isotopes have been found to have isomeric states around the $N=118$ subshell closure [13]. Nuclear structure studies using optical techniques of Fr nuclei were started in the 1980s $[14,15]$ and have recently regained significant interest $[1,4]$. This work presents a continuation of the optical spectroscopy of the ${ }^{206-204} \mathrm{Fr}$ nuclear ground states [1] wherein the isomeric states in the even- $A$ isotopes are under investigation.

\section{EXPERIMENTAL TECHNIQUE}

The experiment was performed at the radioactive-beam facility ISAC (isotope separator and accelerator) at TRIUMF, Vancouver, Canada. Francium isotopes were produced by nuclear reactions in a $\sim 2000^{\circ} \mathrm{C}$ hot $\mathrm{UC}_{x}$ target initiated by a $9.8 \mu \mathrm{A}, 480 \mathrm{MeV}$ proton beam. Subsequent ionization took place within a rhenium-coated transfer tube acting as a surface ion source. Positive ions were then accelerated to $20 \mathrm{keV}$, passed through a high-resolution, two-stage mass separator before being delivered to the experimental setup.

The continuous ion beam was injected into an out-ofplane, gas-filled, radio-frequency quadrupole (RFQ) Paul trap [16] and ejected in reverse direction into the collinear laser spectroscopy beamline [17]. The injection time was adjusted for each nuclear state under investigation and varied between 5 and $500 \mathrm{~ms}$. After a $1 \mathrm{~ms}$ delay, the ions were ejected within a $100 \mu \mathrm{s}$ window for all states. The ion bunches were neutralized in a $\mathrm{Rb}$ vapor cell with $50 \%$ efficiency into the atomic ground state of the Fr atom.

The atoms were shifted into resonance with a counterpropagating laser beam at $718 \mathrm{~nm}$ allowing high-resolution spectroscopy to be performed on the atomic $7 s^{2} S_{1 / 2} \rightarrow$ $7 p^{2} P_{3 / 2}^{\circ}$ transition in neutral Fr. Fluorescent photons were detected in the light collection region, located $40 \mathrm{~cm}$ downstream of the charge exchange cell, using a red-sensitive photomultiplier tube (PMT). The PMT output was recorded 
using a digital multichannel scaler (MCS) data acquisition system with respect to both Doppler tuning voltage and time post RFQ extraction. The latter was implemented by utilizing the RFQ ejection trigger as the start of data accumulation. Long-term frequency stabilization and locking was achieved by comparing a small fraction of the output from an M Squared SolsTiS Ti:Sa laser (pumped by a Lighthouse Sprout Nd:YAG laser) to a frequency-stabilized HeNe laser via a scanning, confocal Fabry-Pérot etalon.

The majority of the Ti:Sa laser output was passed through an electro-optical modulator (EOM) to rapidly modulate the intensity of laser light injected into the beamline and hence minimize the amount of optical pumping in the Fr atoms [1]. A pulse train of transmitted laser light was observed in the light collection region and consisted of a $30 \mathrm{~ns}$ pulse duration with both an $18 \mathrm{~ns}$ rise and fall time at a repetition rate of $333 \mathrm{kHz}$. A time-averaged power of $10 \mathrm{pW}$ corresponded to $1.6 \mathrm{~mW}$ on peak. The transit time from the charge exchange cell to the light collection region of the atom bunch is approximately $3 \mu \mathrm{s}$ at $20 \mathrm{keV}$ corresponding to 140 lifetimes of the $P_{3 / 2}^{\circ}$ state ( $\tau=21 \mathrm{~ns}$ [18]) in Fr. The pulse train therefore corresponds to each atom experiencing one photon burst on average.

Increasing the frequency of the intensity modulation to excite more atoms in close proximity to the PMT begins to re-introduce the kind of optical pumping effect (upstream) that the method was developed to avoid. A new technique has therefore been developed as part of this work whereby each section of the atom bunch was probed by three different laser frequencies, supplied by sequential light pulses. This enhances the effective scanning rate whilst minimizing optical pumping and is reported here for the first time. This was accomplished utilizing the light of the first order diffraction fringe from a double-passed acousto-optical modulator (AOM). The radio-

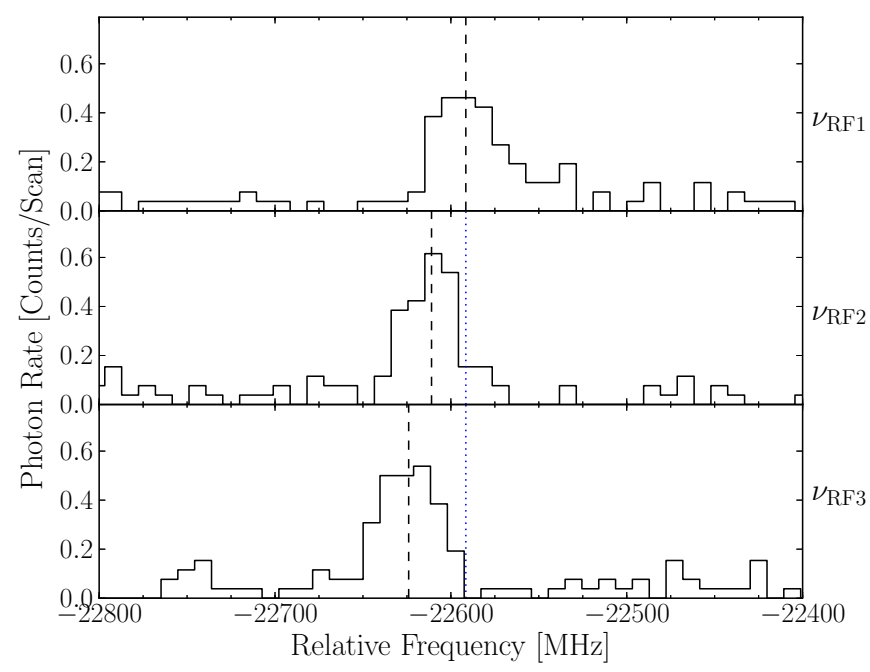

FIG. 1. (Color online) Effect of the different AOM radio frequen$\operatorname{cies}\left(v_{\mathrm{RF} 1}=110, v_{\mathrm{RF} 2}=100\right.$, and $\left.\nu_{\mathrm{RF} 3}=90 \mathrm{MHz}\right)$ illustrated on the cycling transition of ${ }^{208} \mathrm{Fr}$. The spectra for the three different RFs are presented individually. The difference in resonance centroids as indicated by the dashed vertical lines corresponds to the RF offset. As an example, the blue dotted lines correspond to the centroid for $\nu_{\mathrm{RF} 1}$. frequencies (RFs) applied to the AOM are well defined and change the carrier laser frequency by $2 \nu_{\mathrm{RF}}$. Consequently, any individual atom can at most be on resonance with one of the three laser frequencies used, thus maintaining the effectiveness of the intensity modulation.

The high voltage pulses supplied to the EOM to achieve the best distinction and the RF applied to the AOM were synchronized by a delay generator such that one AOM frequency coincided with a photon burst. A coincidence unit was employed to feed the PMT signal for the individual photon bursts, containing different effective laser frequencies, into separate data acquisition (DAQ) channels (see Fig. 1). The resultant three fluorescent spectra are treated individually in the analysis.

\section{RESULTS}

The observed fluorescence spectra for ${ }^{208,206-204} \mathrm{Fr}$ are shown in Fig. 2. For both ${ }^{204,206} \mathrm{Fr}$, three nuclear states were observed. Henceforth, the naming scheme presented in [13] will be used to refer to the individual nuclear states. For ${ }^{206} \mathrm{Fr}$, this marks the first direct and isolated observation of the presence of two long-lived isomeric states. It is noteworthy that the high-frequency (low-spin) multiplet for the nuclear ground states of ${ }^{204 g} \mathrm{Fr}$ and ${ }^{206 g} \mathrm{Fr}$ forms a doublet structure with the respective first, lowest in energy, isomeric states, ${ }^{204 m 1} \mathrm{Fr}$ and ${ }^{206 m} \mathrm{~m} 1 \mathrm{Fr}$.

The data were fitted using various nuclear spins $I$ with the standard hyperfine function whereby the frequency of each allowed hyperfine transition $F \rightarrow F^{\prime}$ is given as

$$
v_{F, F^{\prime}}=v_{J, J^{\prime}}+\left(\alpha_{F^{\prime}} A_{F^{\prime}}+\beta_{F^{\prime}} B_{F^{\prime}}\right)-\left(\alpha_{F} A_{F}+\beta_{F} B_{F}\right)
$$

for a transition between two levels of atomic spin $J, J^{\prime}$. The coefficients $\alpha$ and $\beta$ are composed of spin couplings including the nuclear spin $I$, the atomic spin $J$, and the coupling of the two to the total spin of the system $F$ as

$$
\alpha=\frac{K}{2}, \quad \beta=\frac{B}{4} \frac{\frac{3}{2} C(C+1)+2 I J(I+1)(J+1)}{I J(2 I-1)(2 J-1)},
$$

with $C=F(F+1)-J(J+1)-I(I+1)$. The hyperfine coefficients $A$ and $B$ are related to the nuclear magnetic dipole moment $\mu$ and the spectroscopic electric quadrupole moment $Q_{s}$ as

$$
A=\frac{\mu B_{J}(0)}{I J}, \quad B=e Q_{s}\left\langle V_{z z}\right\rangle,
$$

where $B_{J}(0)$ and $\left\langle V_{z z}\right\rangle$ describe the electromagnetic field and its gradient at the site of the nucleus, respectively. From Eqs. (1) and (2) it is immediately clear that the extraction of nuclear properties with any degree of confidence can only be accomplished if the nuclear spin either is known or can be uniquely determined from the optical spectra observed.

A direct and firm spin determination for the nuclear states under investigation is possible due to the high-resolution spectroscopy being performed leading to the individual hyperfine structure transitions being resolved. The hyperfine multiplets can be paired to form a complete hyperfine spectrum for a given nuclear state by means of the common splitting present in both multiplets. Since the value of such a splitting varies for each nuclear state due to differences in nuclear spins and 


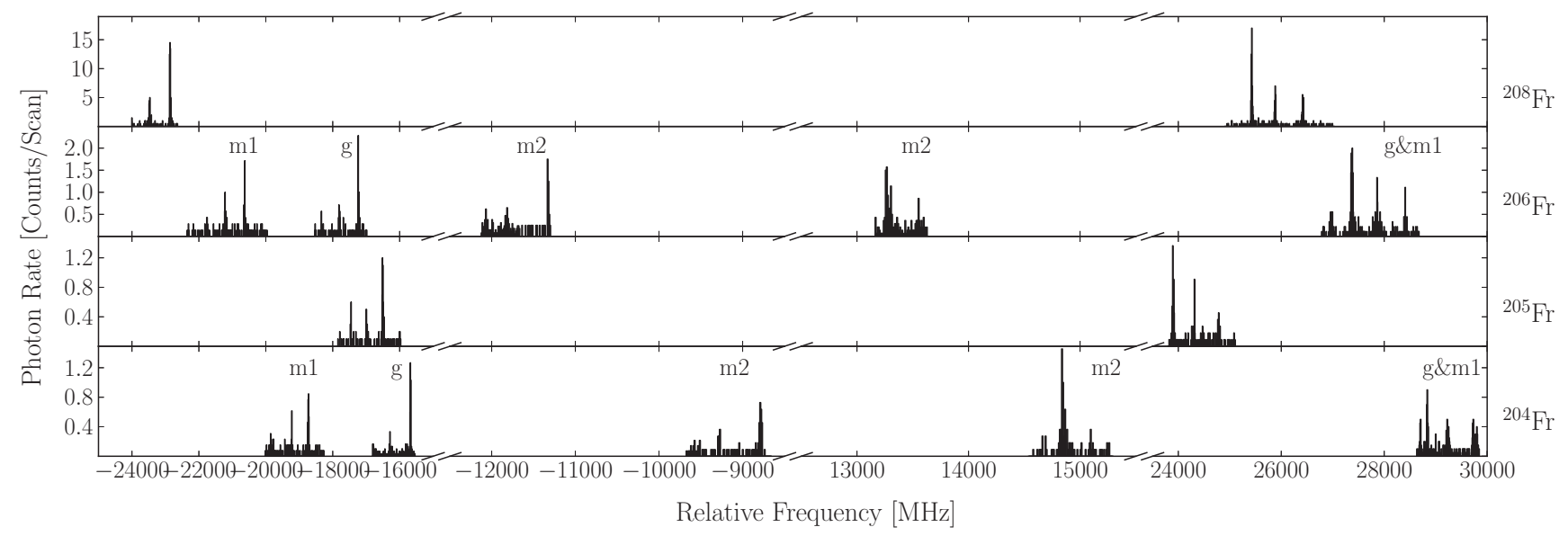

FIG. 2. Observed optical fluorescence spectra for ${ }^{208,206,205,204} \mathrm{Fr}$, respectively. Where appropriate, contributions of the nuclear ground and isomeric states have been marked with a $g$ and $m 1, m 2$, respectively. As discussed in [1], the high-frequency (low-spin) hyperfine multiplets for both ${ }^{206 g, m 1} \mathrm{Fr}$ and ${ }^{204 g, m 1} \mathrm{Fr}$ are doublet structures.

thus angular momentum couplings, the pairing of hyperfine multiplets is unique and unambiguous even in the case of similarly sized nuclear electromagnetic moments. The spin determinations are based upon the goodness of fit value, the observed relative intensities, and the ratio of hyperfine $A$ coefficients [1]. The nuclear spins of the isomeric states for ${ }^{206 m 1,204 m 1} \mathrm{Fr}$ and ${ }^{206 m 2,204 m 2} \mathrm{Fr}$ were determined to be $7^{(+)}$and $10^{(-)}$, respectively. In the case of ${ }^{204 m 1, m 2} \mathrm{Fr}$, the difference in nuclear spin of $\Delta I=3$ is supported by the $L / K$ ratio from recoil-tagged conversion electron spectroscopy [19]. The tentative spin assignments from $\alpha$-decay spectroscopy [13] are herewith confirmed. The resultant nuclear spins for all investigated nuclear states as well as the parameters for the isotope shifts $\delta v^{A, 208}$ and hyperfine coupling constants for both the $S_{1 / 2}$ and $P_{3 / 2}^{\circ}$ atomic states are shown in Table I. The relative intensities closely match the spin-dependent model values as a result of the intensity modulation technique.

The nuclear magnetic dipole moments were extracted by comparing the fitted values for the magnetic hyperfine coupling constant $A$ of the isotope to that of a reference isotope whose magnetic dipole moment $\mu\left({ }^{210} \mathrm{Fr}\right)=+4.38(5) \mu_{\mathrm{N}}$ has been determined from atom trapping experiments [20]. Combined with the hyperfine parameters presented in [15] and in the absence of hyperfine anomalies [21], the magnetic dipole moment of the nuclear states under investigation was determined via

$$
\mu=\frac{I A}{I_{210} A_{210}} \mu_{210} .
$$

Since the hyperfine $A$ parameter was determined for two atomic states, the dipole moments presented in Table I are a weighted mean of those calculated from both atomic states for a given nuclear state.

Since there is no direct measurement of an electric quadrupole moment within the francium isotopic chain, the electromagnetic fields have to be evaluated theoretically in order to extract the nuclear moment. Many-body calculations

TABLE I. Overview of the spins, isotope shifts, and hyperfine coupling constants for the $7 s^{2} S_{1 / 2} \rightarrow 7 p^{2} P_{3 / 2}^{\circ}$ atomic transition in Fr. Changes in mean-squared charge radii $\delta\left\langle r^{2}\right\rangle^{A, 208}$ as well as the nuclear magnetic dipole moment $\mu$ and the spectroscopic electric quadrupole moment $Q_{s}$ were extracted. The uncertainty of the calculation of the electric field gradient at the site of the nucleus is not included in the given uncertainty. An additional $1 \%$ error on $\delta\left\langle r^{2}\right\rangle^{A, 208}$ arises from the theory of atomic factors [23]. These values update and correct earlier values from [1] where systematic errors were unaccounted for. Information on the half-lives and excitation energies are taken from [25] and [13], respectively.

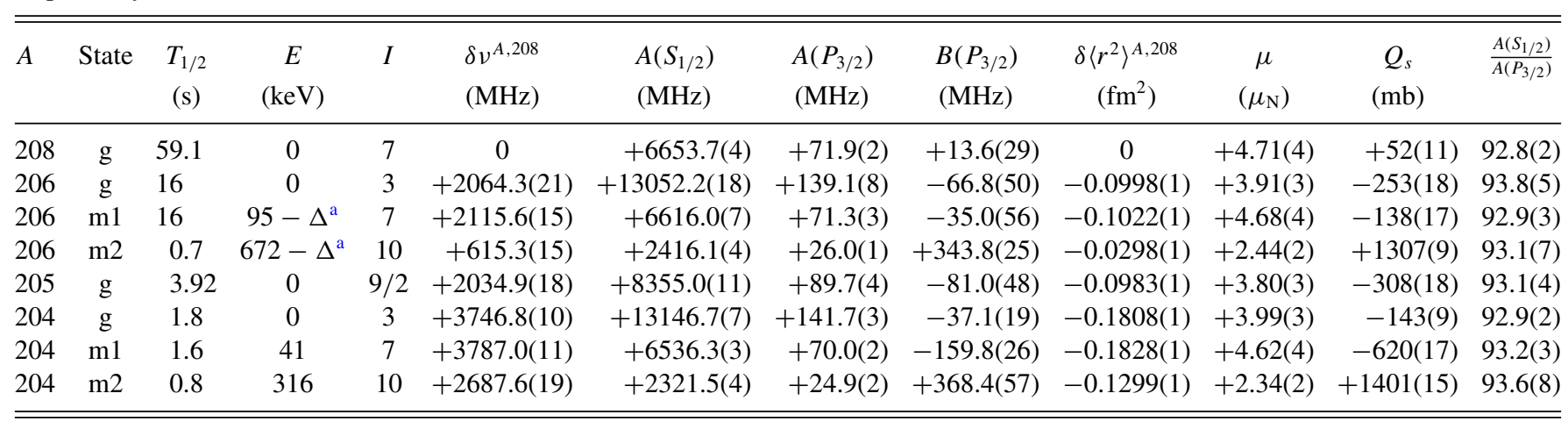

${ }^{\mathrm{a}}$ Represents upper limit as discussed in [13]. 
[15] yield

$$
\frac{B}{Q_{s}}=263 \mathrm{MHz} / \mathrm{b},
$$

allowing the determination of the spectroscopic quadrupole moments presented in Table I. It should be noted, however, that the error given there is purely statistical and does not include any systematic uncertainty because of the unavailability of uncertainties on $B / Q_{s}$.

Changes in mean-squared charge radii $\delta\left\langle r^{2}\right\rangle^{A, 208}$ with respect to ${ }^{208} \mathrm{Fr}$ were extracted from the isotope shifts using

$$
\delta v^{A A^{\prime}}=K\left(\frac{1}{m_{A}}-\frac{1}{m_{A^{\prime}}}\right)+F \delta\left\langle r^{2}\right\rangle^{A A^{\prime}},
$$

where the atomic masses were taken from the latest atomic mass evaluation [22] and the atomic factors,

$$
\begin{aligned}
& F=-20.766(208) \mathrm{GHz} / \mathrm{fm}^{2}, \\
& K=-85(113) \mathrm{GHz} \mathrm{amu},
\end{aligned}
$$

from atomic theory [23]. These calculations have been corroborated by atom trapping techniques [24].

\section{DISCUSSION}

\section{A. Charge radii}

Changes in mean-squared charge radii with respect to $N=$ $126\left({ }^{213} \mathrm{Fr}\right)$ are shown in Fig. 3. The systematic uncertainty according to the atomic factors is of the order of $1 \%$ and shown as the shaded area for the ground states or error bars for the isomeric states. Isodeformation lines from the droplet model $[5,6]$ are included for comparison using $\beta_{2}\left({ }^{213} \mathrm{Fr}\right)=+0.008$ [26].

The odd-even staggering as observed in ${ }^{207-213} \mathrm{Fr}$ [12] continues to lower masses with a more pronounced effect compared to nuclei in the vicinity of the $N=126$ shell closure. For the nuclear ground states, a deviation from the $\beta_{2}=0$ line of zero quadrupole deformation is observed at $N \leqslant 119$ indicating the loss of a perfectly spherical system. Furthermore, it is apparent that the very low lying, $7^{(+)}$ isomeric states follow the trend of the nuclear ground states. An isomeric shift between the two states is of the order of $+50 \mathrm{MHz}$ resulting in $\delta\left\langle r^{2}\right\rangle^{m 1, g}=-0.002 \mathrm{fm}^{2}$ being negligible on the scale presented here.

The second, $10^{(+)}$isomeric states exhibit an isomeric shift from the ground states of $-1449.0(36) \mathrm{MHz}$ for ${ }^{206} \mathrm{Fr}$ and $-1059.2(29) \mathrm{MHz}$ for ${ }^{204} \mathrm{Fr}$. This shift corresponds to a charge radius enlarged compared to that of the ground states. Both masses point to that nuclear state having a root-mean-square deformation of $\left|\beta_{2}\right| \approx 0.10$. While the $\delta\left\langle r^{2}\right\rangle^{m, g}$ is greater for ${ }^{206} \mathrm{Fr}$ than ${ }^{204} \mathrm{Fr}$ (by approximately a factor of 1.4 ) the results for ${ }^{206 m 2} \operatorname{Fr}\left(\left|\beta_{2}\right|>0.15\right)$ as presented in [4] were not confirmed.

A comparison of nuclear ground states between the isotopic chains of Fr and $\mathrm{Pb}$ is given in Fig. 3(a). The correspondence in OES down to $N=119$ is remarkable albeit it is more pronounced for the lower neutron numbers. This is evidenced by the relative change in $\delta\left\langle r^{2}\right\rangle$ for the ground state isotones in Fr and $\mathrm{Pb}$ highlighted in Fig. 3(b). While generally $\delta\left\langle r^{2}\right\rangle$ is

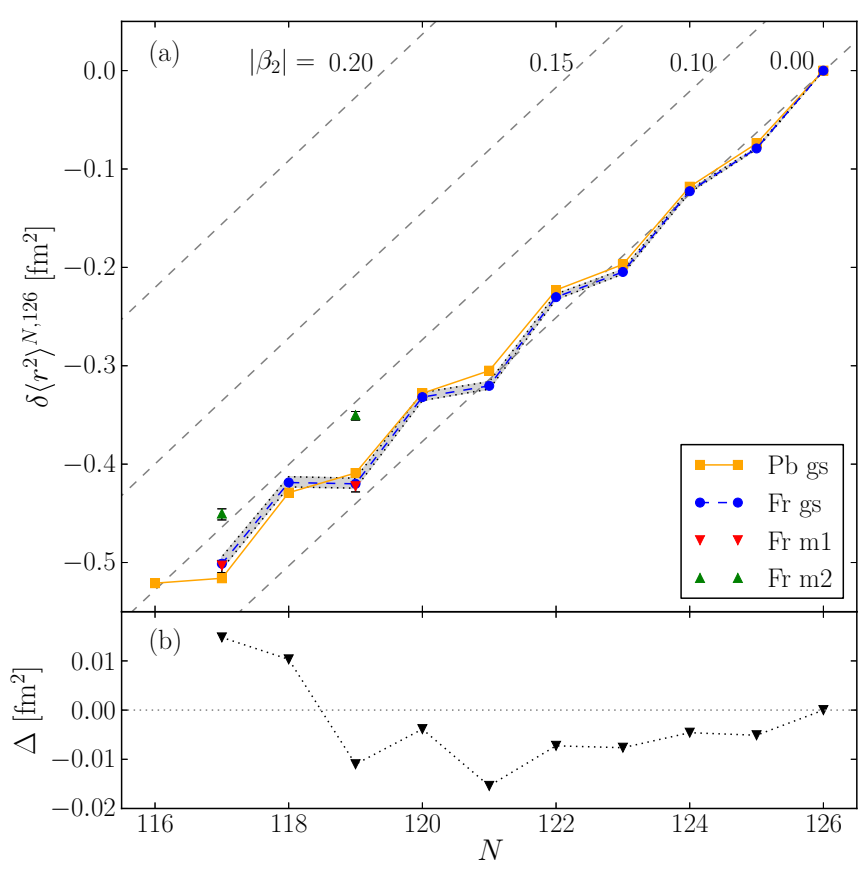

FIG. 3. (Color online) (a) Changes in mean-squared charge radii for the neutron-deficient $\mathrm{Fr}$ isotopes in comparison to those of $\mathrm{Pb}$ $[15,27]$. Statistical errors are within the data points. Systematic errors for Fr are included as the shaded area for the nuclear ground states and error bars for the isomers. Isodeformation lines according to the droplet model [5,6] are shown as the dashed lines using $\beta_{2}\left({ }^{213} \mathrm{Fr}\right)=$ +0.008 from [26]. The shaded area enclosed by the dotted lines corresponds to the systematic uncertainty from the atomic factors. (b) Relative change in $\delta\left\langle r^{2}\right\rangle$ for the $\mathrm{Fr}$ and $\mathrm{Pb}$ ground states; $\Delta=$ $\delta\left\langle r^{2}\right\rangle_{\mathrm{Fr}}^{N, 126}-\delta\left\langle r^{2}\right\rangle_{\mathrm{Pb}}^{N, 126}$.

smaller for Fr than for the $\mathrm{Pb}$ isotone, this trend is reversed at $N=118$ such that the Fr isotopes cease to change as rapidly as their $\mathrm{Pb}$ isotones. Reflected in the reversal of sign in $\delta\left\langle r^{2}\right\rangle_{N}^{\mathrm{Fr}, \mathrm{Pb}}$, the observed behavior marks the loss of close correspondence between the $\mathrm{Fr}$ and $\mathrm{Pb}$ isotones. The changes in mean-squared charge radii therefore indicate that the nuclear ground states are governed by spherical structures with an onset of collectivity at $N=118$.

\section{B. Dipole moments}

The nuclear magnetic dipole moment is linked to the nuclear $g$ factor via $g=\mu / I$ where $I$ is the spin of the corresponding nuclear state. Nuclear $g$ factors provide strong fingerprints for the specific configurations. A change in configuration becomes apparent as a sudden increase or decrease in the $g$ factor. This is of particular importance when isomeric states are present that may occupy different nuclear orbitals. As a comparison to the experimental data, empirical $g$ factors may aid in discussing nucleon orbital occupations by using single-particle coupling estimates and the additivity relation [28]

$$
g_{\text {emp }}=\frac{1}{2}\left[\left(g_{\pi}+g_{\nu}\right)+\left(g_{\pi}-g_{\nu}\right) \frac{j_{\pi}\left(j_{\pi}+1\right)-j_{v}\left(j_{v}-1\right)}{I(I+1)}\right]
$$




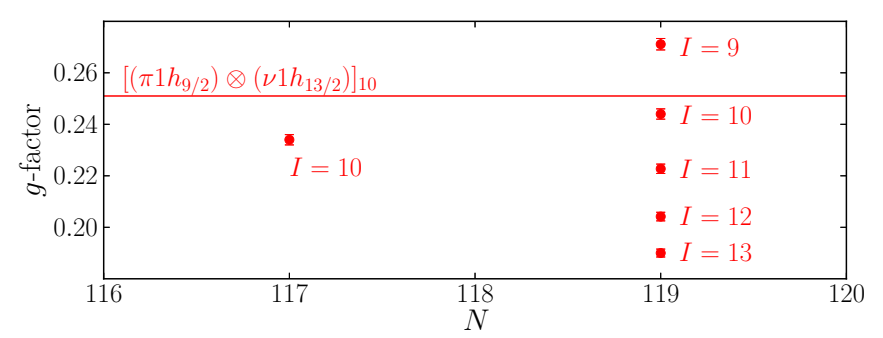

FIG. 4. (Color online) Nuclear $g$ factors for ${ }^{204 m 2,206 m 2} \mathrm{Fr}$ compared to the empirical coupling for a $\left[\left(\pi 1 h_{9 / 2}\right) \otimes\left(\nu 1 i_{13 / 2}\right)\right]_{10}$ configuration (horizontal line). The filled circles for $N=119$ indicate the $g$ factor derived from the fits of hyperfine function to the data for various spins.

for the even- $A$ isotopes, where $g_{\pi}, g_{\nu}$ are the corresponding $g$ factors, $j_{\pi}, j_{v}$ the angular momenta of the valence particles, and $I$ the total nuclear spin. In order to obtain as pure a shell effect as possible and hence to compare to single-particle-like structures, $g_{\pi}, g_{v}$ were evaluated from isotopes with one valence particle outside otherwise closed shells. The corresponding nuclear magnetic dipole moments were taken from [29]. ${ }^{209} \mathrm{Bi}$ with one proton in addition to the doubly magic ${ }^{208} \mathrm{~Pb}$ core was taken to evaluate $g_{\text {emp }}(\pi)=+0.91$. The neutron $g$ factors were taken from $\mathrm{Pb}$ isotopes to avoid any correlating effects between the nucleons. For the $v 3 p_{1 / 2}, v 2 f_{5 / 2}, v 3 p_{3 / 2}$, and $v 1 i_{13 / 2}$ configurations, ${ }^{207,201,197,195} \mathrm{~Pb}$ served as empirical values resulting in $g_{\text {emp }}(v)=+1.19,+0.27,-0.72,-0.17$, respectively.

Considering the specific case of ${ }^{206 m 2} \mathrm{Fr}$, the $\left[\left(\pi 1 h_{9 / 2}\right)^{1} \otimes\right.$ $\left.\left(v 1 i_{13 / 2}\right)^{-1}\right]_{10}$ shell-model state is suggested [30]. Based on the evaluated empirical nuclear $g$ factors for a variety of spins a convincing argument for $I=10$ can be presented. From Fig. 4 it is quite clear, that $I=10$ provides the closest correspondence of $g$ factor to the suggested configuration when fitting the data with various nuclear spins. Regarding $g\left({ }^{206 m 2} \mathrm{Fr}\right) \approx+0.35$ from [4], a significant systematic error of the order of 0.08 or greater for the experiment presented here can be excluded due to the resolved observation of all six hyperfine transitions and a common splitting for both hyperfine multiplets. The assignment of $I=10$ for this isomeric state can be considered confirmed.

The $g$ factors for the neutron-deficient Fr isotopes and their isomers are presented in Fig. 5. A single valence proton located in the $\pi 1 h_{9 / 2}$ shell determines the structure of the odd- $A$, neutron-deficient $\mathrm{Fr}$ isotopes [12] giving rise to the $I=9 / 2$ nuclear ground state spins. Such a valence proton couples with a valence neutron in the even- $A$ Fr isotopes to form ground state configurations where the neutron orbital occupancy may be taken directly from the shell model.

From the $N=126$ neutron shell closure towards more neutron-deficient isotopes a downward sloping trend in the experimentally determined $g$ factors (and thus a deviation from the $g_{\text {emp }}$ for the respective nuclear states) is observed. Such a departure has been observed in $\mathrm{Bi}$ [31] and $\mathrm{Tl}$ [32] and is generally attributed to core polarization effects within the nucleus. For the isomeric states of lower excitation energy with $I=7$, the trend of decreasing $g$ factors in the

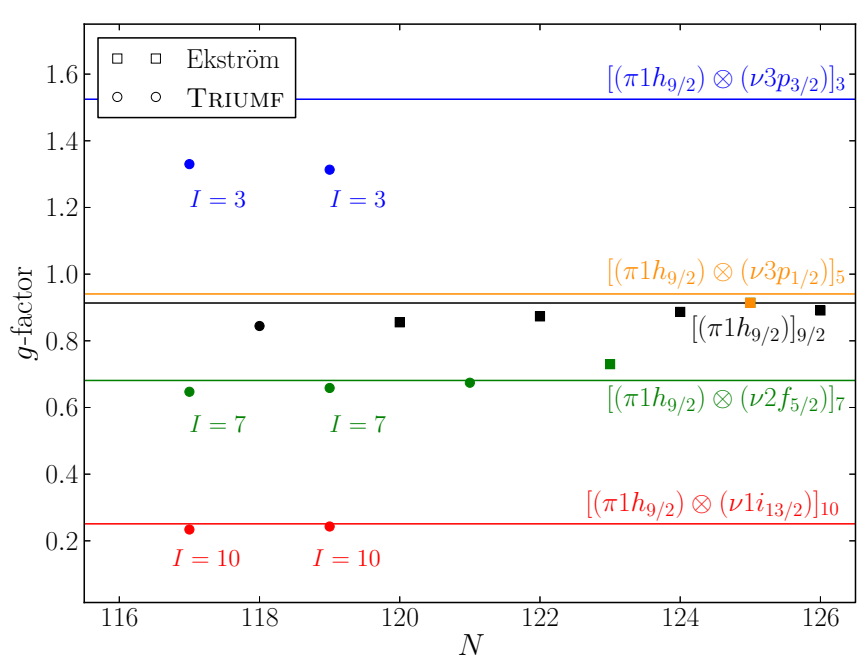

FIG. 5. (Color online) Nuclear $g$ factors for the neutron-deficient Fr isotopes and isomers. The $g$ factors for the relevant valence particle couplings have been determined empirically (see text for details). The data are color-coded with respect to the suggested proton/neutron state occupancy. The values for ${ }^{207,209-213} \mathrm{Fr}$ were taken from [12] (squares) whereas ${ }^{204-206,208} \mathrm{Fr}$ are from the present work (circles).

$v 2 f_{5 / 2}$ shell is continued in ${ }^{206 m 1} \mathrm{Fr}$ and finally ${ }^{204 m 1} \mathrm{Fr}$. The increasing discrepancy of the experimentally and empirically determined $g$ factors indicates an increase in collectivity as the neutron shells are depleted. Deviations from the spherical symmetry imply the strong mixing of spherical shell-model configurations.

\section{Comparison to theoretical values}

Calculations within the energy density functional (EDF) framework were performed utilizing the Skyrme parametrization UNEDF0 [33] and the computer code HFBTHO [34].

TABLE II. Comparison of theoretically determined absolute charge radii $\left\langle r_{\mathrm{ch}}\right\rangle$, binding energies $B$, and intrinsic point-proton quadrupole moments $Q_{0}$ using UNEDF0-EDF for the nuclear ground states. The statistical model errors for the UNEDF0 results were determined using the procedure from [35]. The experimental binding energies were calculated from the binding energies per nucleon [22].

\begin{tabular}{ccccccc}
\hline \hline \multirow{2}{*}{$A$} & \multicolumn{2}{c}{$\begin{array}{c}\left\langle r_{\mathrm{ch}}\right\rangle \\
(\mathrm{fm})\end{array}$} & & \multicolumn{2}{c}{$B$} \\
$(\mathrm{GeV})$ & & $\begin{array}{c}Q_{0} \\
(\mathrm{~b})\end{array}$ \\
\cline { 2 - 3 } & EDF & Expt. & EDF & Expt. & EDF \\
\hline 213 & $5.576(6)$ & 5.577 & $-1.65714(49)$ & $-1.65468(1)$ & $-0.40(8)$ \\
212 & $5.573(6)$ & 5.570 & $-1.64825(52)$ & $-1.64657(1)$ & $-0.60(6)$ \\
211 & $5.567(6)$ & 5.566 & $-1.64046(57)$ & $-1.63912(1)$ & $-0.77(17)$ \\
210 & $5.563(5)$ & 5.559 & $-1.63109(63)$ & $-1.63024(1)$ & $-1.30(15)$ \\
209 & $5.561(5)$ & 5.556 & $-1.62270(73)$ & $-1.62261(1)$ & $-2.03(20)$ \\
208 & $5.560(5)$ & 5.548 & $-1.61336(76)$ & $-1.61344(1)$ & $-2.84(8)$ \\
207 & $5.557(5)$ & 5.547 & $-1.60483(79)$ & $-1.60554(2)$ & $-3.02(14)$ \\
206 & $5.556(5)$ & 5.539 & $-1.59535(81)$ & $-1.59587(3)$ & $-3.76(12)$ \\
205 & $5.554(5)$ & 5.539 & $-1.58686(87)$ & $-1.58787(1)$ & $-4.02(13)$ \\
204 & $5.551(5)$ & 5.532 & $-1.57721(91)$ & $-1.57788(2)$ & $-4.36(12)$ \\
\hline \hline
\end{tabular}


A quasiparticle blocking procedure was employed with an equal filling approximation to calculate the binding energies, absolute charge radii, and intrinsic point-proton quadrupole moments for the ground states as listed in Table II. Since the equal filling approximation forces the solution to have timereversal symmetry, the magnetic dipole moments may not be determined within this framework. The experimental absolute mean charge radii $\left\langle r_{\mathrm{ch}}\right\rangle$ were evaluated using $\left\langle r_{\mathrm{ch}}\right\rangle\left({ }^{212} \mathrm{Fr}\right)=$ $5.570 \mathrm{fm}$ from [21] with any error on that value causing a systematic offset only.

The EDF approach applied to the region of Fr isotopes is seen to have problems describing the ground state properties compared to the data. The model overpredicts the intrinsic deformation of the investigated nuclei, leading to deficiencies in the isotopic trend for the charge radii. Similar effects have been seen by other beyond-mean-field models studying the neutron-deficient $\mathrm{Pb}$ isotopes [36].

\section{CONCLUSION}

Collinear laser spectroscopy was performed on neutrondeficient Fr isotopes and isomers at the ISAC facility at TRIUMF, Vancouver, Canada. The technique of high-frequency intensity modulation previously developed on the nuclear ground states to suppress hyperfine pumping [1,37] was employed to enhance the intensities of the nonstretched atomic hyperfine transitions. Furthermore, the aforementioned technique was refined by means of different effective laser frequencies for each photon burst. As with the technique of intensity modulation, the further development of probing atoms with slightly different velocity classes may be applied to any collinear laser spectroscopic work performed on atoms where branching to other fine or hyperfine structure state is possible.

For the first time, the presence of two isomeric states in ${ }^{206} \mathrm{Fr}$, ${ }^{206 m 1} \mathrm{Fr}$, and ${ }^{206 m 2} \mathrm{Fr}$ was definitively verified. The high-resolution spectroscopy performed in this study allowed an unambiguous pairing of the hyperfine multiplets through the hyperfine splittings despite the overlapping multiplets of ${ }^{206 g, 206 m 1} \mathrm{Fr}$ and ${ }^{204 g, 204 \mathrm{~m} 1} \mathrm{Fr}$. The model-independent determination of the nuclear spin for the isomeric states in ${ }^{206,204}$ Fr allowed a spin-independent extraction of the changes in mean-squared charge radii as well as the magnetic dipole and electric quadrupole moments. The changes in mean-squared charge radii indicate an onset of collectivity at $N=118$.

Calculations within the UNEDF0-EDF framework reflect the binding energies very well at levels below $0.1 \%$; however, deficiencies in predicting other nuclear properties exist due to deformation effects.

\section{ACKNOWLEDGMENTS}

The authors thank J. A. Behr from TRIUMF for fruitful discussions and comments on this manuscript, as well as the FrPNC Collaboration for narrow-frequency laser support. The work presented was financed by the Natural Sciences and Engineering Research Council of Canada (NSERC) under Grant No. 341308-2011, TRIUMF which receives funding from the National Research Council of Canada (NRC), the Sciences and Technology Facilities Council (STFC) of the United Kingdom, and by the Academy of Finland under the Finnish Centre of Excellence Programme 2012-2017 (Project No. 251353, Nuclear and Accelerator-Based Physics Research at JYFL) and the FIDIPRO program. A.V. acknowledges additional support from the Magnus Ehrnrooth Foundation.
[1] A. Voss, M. R. Pearson, J. Billowes, F. Buchinger, B. Cheal, J. E. Crawford, A. A. Kwiatkowski, C. D. P. Levy, and O. Shelbaya, Phys. Rev. Lett. 111, 122501 (2013).

[2] W. Borchers, R. Neugart, E. W. Otten, H. T. Duong, G. Ulm, and K. Wendt (ISOLDE Collaboration), Hyperfine Interact. 34, 25 (1987).

[3] H. De Witte, A. N. Andreyev, N. Barré, M. Bender, T. E. Cocolios, S. Dean, D. Fedorov, V. N. Fedoseyev, L. M. Fraile, S. Franchoo, V. Hellemans et al., Phys. Rev. Lett. 98, 112502 (2007).

[4] K. M. Lynch, J. Billowes, M. L. Bissell, I. Budinčević, T. E. Cocolios, R. P. De Groote, S. De Schepper, V. N. Fedosseev, K. T. Flanagan, S. Franchoo et al., Phys. Rev. X 4, 011055 (2014).

[5] W. D. Myers and K.-H. Schmidt, Nucl. Phys. A 410, 61 (1983).

[6] D. Berdichevsky and F. Tondeur, Z. Phys. A Atoms Nuclei 322, 141 (1985).

[7] J. Bonn, G. Huber, H.-J. Kluge, L. Kugler, and E. W. Otten, Phys. Lett. B 38, 308 (1972).

[8] G. Ulm, S. K. Bhattacherjee, P. Dabkiewicz, G. Huber, H.-J. Kluge, T. Kühl, H. Lochmann, E.-W. Otten, K. Wendt, S. A. Ahmad et al., Z. Phys. A Atomic Nuclei 325, 247 (1986).
[9] Th. Hilberath, St. Becker, G. Bollen, H. J. Kluge, U. Krönert, G. Passler, J. Rikovska, and R. Wyss (ISOLDE Collaboration), Z. Phys. A 342, 1 (1992).

[10] T. E. Cocolios, W. Dexters, M. D. Seliverstov, A. N. Andreyev, S. Antalic, A. E. Barzakh, B. Bastin, J. Büscher, I. G. Darby, D. V. Fedorov et al., Phys. Rev. Lett. 106, 052503 (2011).

[11] E. W. Otten, in Treatise on Heavy Ion Science (Springer, New York, 1989), p. 517.

[12] C. Ekström, L. Robertsson, and A. Rosén, Phys. Scr. 34, 624 (1986).

[13] M. Huyse, P. Decrock, P. Dendooven, G. Reusen, P. Van Duppen, and J. Wauters, Phys. Rev. C 46, 1209 (1992).

[14] S. Liberman, J. Pinard, H. T. Duong, P. Juncar, P. Pillet, J.-L. Vialle, P. Jacquinot, F. Touchard, S. Büttgenbach, C. Thibault et al., Phys. Rev. A 22, 2732 (1980).

[15] A. Coc, C. Thibault, F. Touchard, H. T. Duong, P. Juncar, S. Liberman, J. Pinard, J. Lermé, J. L. Vialle, S. Büttgenbach et al., Phys. Lett. B 163, 66 (1985).

[16] J. Dilling, R. Baartman, P. Bricault, M. Brodeur, L. Blomeley, F. Buchinger, J. Crawford, J. R. C. López-Urrutia, P. Delheij, M. Froese et al., Int. J. Mass Spectrom. 251, 198 (2006).

[17] E. Mané, J. A. Behr, J. Billowes, T. Brunner, M. Brodeur, F. Buchinger, J. E. Crawford, J. Dilling, S. Ettenauer, C. D. P. Levy et al., Hyperfine Interact. 199, 357 (2011). 
[18] W. Z. Zhao, J. E. Simsarian, L. A. Orozco, W. Shi, and G. D. Sprouse, Phys. Rev. Lett. 78, 4169 (1997).

[19] U. Jakobsson, J. Uusitalo, S. Juutinen, M. Leino, T. Enqvist, P. T. Greenlees, K. Hauschild, P. Jones, R. Julin, S. Ketelhut et al., Phys. Rev. C 85, 014309 (2012).

[20] E. Gomez, S. Aubin, L. A. Orozco, G. D. Sprouse, E. IskrenovaTchoukova, and M. S. Safronova, Phys. Rev. Lett. 100, 172502 (2008).

[21] J. S. Grossman, L. A. Orozco, M. R. Pearson, J. E. Simsarian, G. D. Sprouse, and W. Z. Zhao, Phys. Rev. Lett. 83, 935 (1999).

[22] M. Wang, G. Audi, A. H. Wapstra, F. G. Kondev, M. MacCormick, X. Xu, and B. Pfeiffer, Chin. Phys. C 36, 1603 (2012).

[23] V. A. Dzuba, W. R. Johnson, and M. S. Safronova, Phys. Rev. A 72, 022503 (2005).

[24] R. Collister, G. Gwinner, M. Tandecki, J. A. Behr, M. R. Pearson, J. Zhang, L. A. Orozco, S. Aubin, and E. Gomez (FrPNC Collaboration), Phys. Rev. A 90, 052502 (2014).

[25] J. K. Tuli, Nuclear Wallet Cards, National Nuclear Data Center, Brookhaven National Laboratory, 2011.

[26] P. Moller, J. R. Nix, W. D. Myers, and W. J. Swiatecki, At. Data Nucl. Data Tables 59, 185 (1995).

[27] M. Anselment, W. Faubel, S. Göring, A. Hanser, G. Meisel, H. Rebel, and G. Schatz, Nucl. Phys. A 451, 471 (1986).
[28] R. J. Blin-Stoyle, Rev. Mod. Phys. 28, 75 (1956).

[29] N. J. Stone, IAEA Report No. INDC(NDS)-0594, 2011.

[30] F. G. Kondev, Nucl. Data Sheets 109, 1527 (2008).

[31] M. R. Pearson, P. Campbell, K. Leerungnavarat, J. Billowes, I. S. Grant, M. Keim, J. Kilgallon, I. D. Moore, R. Neugart, M. Neuroth et al., J. Phys. G: Nucl. Part. Phys. 26, 1829 (2000).

[32] A. E. Barzakh, L. Kh. Batist, D. V. Fedorov, V. S. Ivanov, K. A. Mezilev, P. L. Molkanov, F. V. Moroz, S. Yu. Orlov, V. N. Panteleev et al., Phys. Rev. C 88, 024315 (2013).

[33] M. Kortelainen, T. Lesinski, J. Moré, W. Nazarewicz, J. Sarich, N. Schunck, M. V. Stoitsov, and S. Wild, Phys. Rev. C 82, 024313 (2010).

[34] M. V. Stoitsov, N. Schunck, M. Kortelainen, N. Michel, H. Nam, E. Olsen, J. Sarich, and S. Wild, Comput. Phys. Commun. 184, 1592 (2013).

[35] M. Kortelainen, J. Erler, W. Nazarewicz, N. Birge, Y. Gao, and E. Olsen, Phys. Rev. C 88, 031305(R) (2013).

[36] J. M. Yao, M. Bender, and P.-H. Heenen, Phys. Rev. C 87, 034322 (2013).

[37] T. J. Procter, J. A. Behr, J. Billowes, F. Buchinger, B. Cheal, J. E. Crawford, J. Dilling, A. B. Garnsworthy, A. Leary, C. D. P. Levy et al., Eur. Phys. J. A 51, 23 (2015). 